\title{
Management of borderline personality disorder
}

\author{
Robert S. Biskin MD, Joel Paris MD
}

See related review article by Biskin and Paris on diagnosis of borderline personality disorder at www.cmaj.ca/lookup/doi/10.1503/cmaj.090618

$\mathrm{B}$ orderline personality disorder is characterized by intense, rapidly fluctuating moods combined with impulsivity and interpersonal difficulties. Patients with the disorder are frequently encountered in clinical practice, despite a prevalence in the community of $1 \%-2 \% .{ }^{1}$ Up to $10 \%$ of patients seen in outpatient psychiatry clinics ${ }^{2,3}$ and $6 \%$ of those seen in a family medicine clinic ${ }^{4}$ meet criteria on diagnostic interviews, although rates are much higher and more varied when self-report measures are used. ${ }^{5,6}$ In general, patients show a gradual improvement in symptoms with age, ${ }^{7}$ although functioning remains impaired. ${ }^{8}$ One long-term community-based follow-up study found a $10 \%$ suicide rate. ${ }^{9}$

The management of patients with borderline personality disorder is often challenging, with substantial risks of inappropriate or insufficient treatment. In this article, we review the literature and provide a practical and evidence-based approach to the management of borderline personality disorder. A summary of the evidence used in this review is found in Box 1. We based our review primarily on findings from randomized controlled trials and meta-analyses. We included results from other types of studies for topics where no controlled trials were available.

A related review article on the diagnosis of borderline personality disorder is available at www.cmaj.ca/lookup/doi/10.1503/cmaj.090618.

\section{Is admission to hospital helpful?}

Patients with borderline personality disorder are often initially seen in the emergency department, frequently because of self-harm, suicidal thoughts or a suicide attempt. These episodes often occur following acute psychosocial stressors. The first and most pressing question to consider is whether hospital admission is required. Many experts suggest that admission is often unproductive or even counterproductive and should be avoided. ${ }^{10-12}$ Serious suicide attempts and acute psychotic symptoms may warrant admission, but clinical experience suggests that brief stays are preferable. ${ }^{12}$
Patients should not be admitted to hospital because admission may result in a regression in unhealthy behaviour such as passivity, drug or alcohol abuse and acting out, ${ }^{13}$ or disruptive behaviour such as self-harm, violence and rule violations. ${ }^{14,15} \mathrm{~A}$ rapid outpatient psychiatric intervention or a day-hospital setting are better choices and prevent the loss of potentially stabilizing social contacts and employment. Although admission to a general psychiatric ward is not recommended in most instances, several specialized inpatient treatments for borderline personality disorder have been shown to be efficacious ${ }^{16,17}$ and may be excellent choices if available.

Minor suicidal gestures or threats can often be managed on an outpatient basis or with an overnight stay in the emergency department to allow the patient to "cool off" and reappraise his or her current situation. Some experts suggest that therapeutic interventions provided in the emergency department should be primarily concrete and supportive, with more in-depth psychotherapeutic work limited to the patient's regular treatment and provided after the emotional dysregulation has passed. ${ }^{10,18}$ Psychosocial interventions or referrals to more appropriate treatments can then be arranged at discharge the following morning.

\section{What is the primary treatment?}

Psychotherapy is the most important component in the treatment of borderline personality disorder, leading to large reductions in symptoms that
Competing interests: None declared.

This article has been peer reviewed.

Correspondence to: Robert S. Biskin, robert.biskin@mcgill.ca

CMAJ 2012. DOI:10.1503 /cmaj.112055

\section{- Ker POINTS}

- Borderline personality disorder can be treated successfully, with reductions in self-harm, suicidality, impulsivity and other symptoms.

- Treatment on an outpatient basis or in a day-hospital program is preferable to hospital admission when possible.

- Specialized psychotherapy is the primary form of treatment and is supported by the strongest evidence.

- Pharmacotherapy is supported by less high-quality evidence but may be beneficial as an adjunct to psychotherapy. 
persist over time. Over the past 2 decades, many forms of psychotherapy have been developed specifically to treat the disorder. The best studied are dialectical behaviour therapy, mentalization-based treatment, transference-focused psychotherapy, and systems training for emotional predictability and problem solving. In this article, we focus on dialectical behaviour therapy and mentalization-based treatment, two of the most popular and oldest specialized treatments of borderline personality disorder. Other forms of psychotherapy, including transferencefocused psychotherapy and systems training for emotional predictability and problem solving, can be helpful, and the evidence supporting their use is summarized in Appendix 1 (available at www.cmaj.ca/lookup/suppl/doi:10.1503/cmaj $.112055 /$ //DC1).

Many studies have shown the benefits of the specialized psychotherapy techniques, although problems with both the research methodology and applicability of the results highlight the need for further research. One of the concerns is that, although many techniques have high-quality studies supporting their use, rarely is there more than 1 study. A second concern is that many studies used frequency of visits to the emergency department, incidents of self-harm and suicidality as primary outcome measures. This approach lessens the focus on many of the less dramatic symptoms of the disorder and, more important, ignores psychosocial functioning and overall quality of life. A summary table of randomized controlled trials of psychotherapies for borderline personality disorder is available in Appendix 2 (at www.cmaj.ca/lookup/suppl/doi:10.1503/cmaj $.112055 /-/ D C 1)$.

In practice, the specialized psychotherapy techniques are often not available to patients because they require teams of therapists who have all received the necessary specialized training, which is available only in a small number of university centres and may be extensive. Also, most

\section{Box 1: Evidence used in this review}

We searched Ovid MEDLINE for articles published from 1948 to April 2011. The search term "borderline personality disorder" was combined with "psychotherapy" or "drug therapy." This combination produced 1276 references, of which 1071 were in English. We first limited the search to randomized controlled trials (RCTs) and reviewed the titles and abstracts of 74 articles, 68 of which were deemed to be relevant. We then searched for meta-analyses by limiting the search term "borderline personality disorder" to meta-analyses; we reviewed the titles and abstracts of 16 identified references, of which 4 were included. If either of us was aware of other relevant studies, these were also included. Because study methodology, diagnostic methods, interventions and outcome measures differed greatly between the RCTs, we could not pool the results in a meta-analysis. For topics that were not evaluated in RCTs, we examined findings from other types of studies. of these psychotherapies require treatment for a year or longer, which may be costly.

Because a number of psychotherapy techniques have been shown to be efficacious in the treatment of borderline personality disorder, comparisons between these treatments can be considered. Few studies have been conducted, and in general, there are few clinically important differences in outcomes favouring 1 treatment over another. ${ }^{19,20}$ Similarly, when these treatments are compared with other, highly structured and manualized treatments, differences diminish, although patients in all groups improve dramatically. This suggests that common factors explain part of the efficacy of treatment, with structure being particularly important. ${ }^{21} \mathrm{~A}$ clearer understanding of what parts of these complex interventions are most important may lead to refinements of treatment and potential cost savings. The most recent Cochrane review of psychotherapy for borderline personality disorder, although it included only studies published to 2003, found support for dialectical behaviour therapy and mentalization-based treatment, ${ }^{22}$ and recent findings strengthen this conclusion.

\section{Dialectical behaviour therapy}

Dialectical behaviour therapy was the first psychotherapy shown to be effective specifically in the treatment of borderline personality disorder. ${ }^{23}$ This manualized therapy combines cognitive behavioural therapy with Eastern philosophy and traditions. It has a strict hierarchy of treatment targets, with life-threatening behaviours at the top of the list. One of the key dialectics in the treatment is the balance that the therapist must achieve in validating the experiences and behaviours of the patient while promoting change. ${ }^{10}$ The therapy includes weekly individual sessions and weekly life-skills group sessions that teach skills in 4 domains: mindfulness, distress tolerance, regulation of emotions and interpersonal effectiveness. Phone consultation with the therapist is available at all hours, and team consultation meetings play an important role. ${ }^{10}$ The therapy is designed to last at least 1 year; subsequent phases have been suggested but not thoroughly described. ${ }^{10}$

Dialectical behaviour therapy has the most supporting evidence compared with other psychotherapy techniques for the treatment of borderline personality disorder. The first study of its use for patients with the disorder compared the therapy with community treatment as usual. After 1 year, patients in the treatment group $(n=$ 22 ) had an $80 \%$ reduction $(p<0.005$, one-tailed) in suicidal behaviours and fewer inpatient days and were twice as likely to stay in treatment compared with patients in the usual-treatment 
group $(n=22){ }^{23}$ The treatment group also showed improvements in measures of anger, social functioning and global assessment and required fewer psychotropic medications, with between-group effect sizes ranging from 0.57 for self-reported social functioning to 0.81 for anger. ${ }^{24}$ As seen with many pharmacotherapy studies, both groups improved on other ratings: depression, hopelessness and reasons for living. ${ }^{23}$ The cohort was followed up after 1 year, and the treatment group had improved interviewer-rated social adjustment and no days of inpatient treatment compared with a mean of 5.3 days in the comparison group..$^{25}$

This study has been replicated several times, with consistent results. ${ }^{26,27}$ Linehan and colleagues conducted a second study that compared dialectical behaviour therapy with treatment by community experts. ${ }^{28}$ They found that patients who received dialectical behaviour therapy $(n=$ 52) had half the number of suicide attempts as the comparison group $(n=49)$ (number needed to treat $[\mathrm{NNT}] 4.24,95 \%$ confidence interval [CI] 2.40-18.07]), had fewer admissions to hospital (NNT 3.88, 95\% CI 2.26-13.71) and visits to the emergency department (NNT 9.09, 95\% CI 3.30-12.04) and were more than twice as likely to remain in treatment and be available for follow-up at 1 year.

In another study, dialectical behaviour therapy was compared with general psychiatric management, a highly structured treatment for borderline personality disorder that incorporates weekly individual therapy and medication but no group therapy. ${ }^{29}$ After 1 year, no significant differences in the frequency or severity of suicidal or self-harming behaviours were found between the 2 groups of 90 patients each, nor were there differences in symptoms of borderline personality disorder, other psychiatric symptoms, quality of life, health care utilization or treatment retention.

Length of treatment may be a problem with dialectical behaviour therapy. However, 1 study showed that 13 weeks of life-skills group therapy was superior to standard group therapy. ${ }^{30}$ Another study showed that a shortened, 6-month course of the full program resulted in significant reductions in target behaviours, including nonsuicidal self-injury and suicidal ideation. ${ }^{31}$

Two studies showed dialectical behaviour therapy to be effective in patients with borderline personality disorder who had substance dependence. ${ }^{32,33}$

Overall, dialectical behaviour therapy is an effective treatment for patients with borderline personality disorder. A recent meta-analysis showed that, when compared with usual treat- ment, it yielded lasting benefits, with a moderate effect size of 0.51 (95\% CI $0.38-0.68)$ on global functioning and an effect size of 0.60 for randomized controlled trials (95\% CI 0.49-0.71) on self-harm or suicidal behaviours, even after accounting for different durations of treatment, settings and quality of studies. ${ }^{34}$

\section{Mentalization-based treatment}

Mentalization-based treatment is the second psychotherapy technique developed specifically for the treatment of borderline personality disorder. It has roots in attachment theory, ${ }^{35}$ and its goal is to improve the patient's ability to "mentalize" that is, to understand his or her own and others' mental states. ${ }^{36}$ The treatment uses weekly individual therapy sessions and group sessions over 18 months. It has the advantage of having much shorter training periods and requirements than those for other treatments. ${ }^{18}$

Mentalization-based treatment was first shown to be efficacious in a randomized controlled trial that compared the use of it in a dayhospital setting with standard psychiatric care. ${ }^{37}$ When the patients were followed up at 18 months $(n=44)$ and at 8 years $(n=41)$ after treatment, retention of gains and some continued improvement were observed. ${ }^{38,39}$ By 8 years, mentalization-based treatment showed effect sizes of 1.4 (95\% CI 1.3-1.5) for suicide attempts and 1.8 (95\% CI 0.14-3.50]) for reductions in symptoms, with similar reductions in the use of health care services.

This form of psychotherapy was also found to be effective in an outpatient setting compared with structured clinical management, with an overall odds ratio of 0.28 (95\% CI 0.13-0.61) for hospital admission, suicide and self-injury. ${ }^{36}$

\section{Other forms of psychotherapy}

Other psychotherapy techniques can be effective in the treatment of borderline personality disorder. They include transference-focused psychotherapy, systems training for emotional predictability and problem solving, and standard cognitive behavioural therapy.

Transference-focused psychotherapy is a variant of psychodynamic psychotherapy, often lasting for a year with twice-weekly individual therapy sessions. The primary focus is on emotions arising in the relationship with the therapist and the therapist's use of traditional psychodynamic techniques, such as interpretation. Clear limits and a treatment contract are developed at the beginning of therapy.

Systems training for emotional predictability and problem solving (STEPPS) is a 20 -week group therapy program for patients with border- 
line personality disorder that incorporates elements of cognitive behavioural therapy, skills training and systems therapy. It is intended as an "add-on" treatment for patients being managed in the community.

Evidence supporting the use of these other psychotherapy techniques is outlined in Appendix 1 (available at www.cmaj.ca/lookup/suppl /doi:10.1503/cmaj.112055/-/DC1).

\section{What if specialized psychotherapy is unavailable?}

Nearly all of the psychotherapy techniques we have described are highly specialized and require specially trained therapists working in teams. This limits access to these interventions, particularly in smaller centres or more remote regions. In areas where specialized treatments are available, patients with borderline personality disorder should be referred to these services because data consistently have shown them to be superior to usual treatment.

In areas where specialized programs do not exist, referral to psychotherapists with experience and expertise in the treatment of borderline personality disorder who can provide a structured treatment program may be the best option, but no specific type of psychotherapy is preferable to another in this context. It may be helpful if patients engage in a treatment program that combines multiple modalities with different therapists, but the therapists should work as a team or have contact with each other to avoid conflicting treatment approaches.

\section{Does pharmacotherapy play a role?}

The role of pharmacotherapy in the treatment of borderline personality disorder is limited. The efficacy of different drugs has been studied, but the research methodology limits the applicability of results to clinical practice, for 5 main reasons. First, many of the pharmacotherapy trials were short, often lasting between 6 and 12 weeks. Because the condition is, by definition, a longstanding disorder, it is unclear whether changes that occurred during treatment persisted over time. Second, the sample sizes were small in most of the trials. This was compounded by high dropout rates. The third limitation is the choice of outcome measures. Many studies reported changes in self-reported ratings of depression, anxiety or general psychiatric symptoms, but these measures are not meant to assess changes in symptoms of borderline personality disorder.
Changes attributed to the study medication could therefore have been due to treatment of comorbid disorders or subsyndromal pathology, even if treatment of comorbid depression is more difficult in these patients. ${ }^{40}$ The use of validated measures to assess the effect of treatment on specific symptoms of borderline personality disorder such as self-harm, suicidality or impulsivity would be helpful. The fourth limitation is the number of exclusion criteria. Most patients with borderline personality disorder frequently experience suicidality and often have multiple comorbidities. ${ }^{41}$ Therefore, trials with long lists of exclusions that included suicidal ideation and depression have samples that likely have less severe borderline personality disorder and are less representative of patients seen in practice. The final limitation is the lack of replication. Except for olanzapine, most medications only have 1 or 2 small trials showing their effects. When multiple trials do exist, the studies have often been conducted by the same group of researchers.

Because many patients with borderline personality disorder are prescribed multiple medications ${ }^{42}$ it is important to see which medications have the most evidence. Several meta-analyses of pharmacotherapy trials for borderline personality disorder have shown similar results but have the limitations described above. ${ }^{43-46}$ Because of the paucity of studies assessing medications and the small samples of these studies, some of the meta-analyses pooled medications by drug class, and most of the trials were included despite the methodologic limitations of individual studies. These meta-analyses offer cautious and sometimes conflicting support for the use of medications to partially reduce individual symptoms, but no medications were shown to be effective for the treatment of all domains of symptoms of borderline personality disorder.

In general, meta-analyses have shown that mood stabilizers reduce anger and impulsivity somewhat and may have some effect on affective instability and depression, with standardized mean differences ranging between 0.55 and 1.75 for the different symptoms and in different analyses. ${ }^{44,45}$ Antidepressants may be of modest benefit in reducing anger (standardized mean differences 0.34 and 0.37 ) but have little or conflicting benefit in the treatment of depressive symptoms or impulsivity. ${ }^{44,45}$ Finally, antipsychotic agents were found to have a moderate effect in reducing cognitive symptoms and anger, with effect sizes of 0.56 to $0.63,{ }^{45}$ but more recent findings for olanzapine in particular ${ }^{47}$ were not included in the meta-analyses. The most recent Cochrane review of pharmacotherapy for borderline personality 
disorder highlighted the limitations in this literature but offered some support for mood stabilizers, second-generation antipsychotic agents and omega-3 fatty acids. However, it warned that these recommendations were based almost exclusively on single, small, brief studies and that no medications were effective in treating the core pathology of borderline personality disorder..$^{48}$ Appendix 3 (available at www.cmaj.ca/lookup /suppl/doi:10.1503/cmaj.112055/-/DC1) outlines the evidence for the use of specific antipsychotics, mood stabilizers, antidepressants and other medications in the treatment of borderline personality disorder. A summary table of randomized controlled trials of medications used to treat the disorder is available in Appendix 2.

Pharmacotherapy for borderline personality disorder should be based on symptoms and be offered with concomitant psychotherapy. The data do not provide guidance in choosing medications within drug classes and focus only on reducing symptoms that are associated with the disorder, with no medications producing remission. ${ }^{49}$ As well, the risk of overdose and burden of adverse effects of many of these medications can be substantial, with weight gain and metabolic changes an underrecognized concern, because many of the studies may be too short to show these problems. In practice, when choosing medications, clinicians should balance the potential long-term burden of adverse effects with the knowledge of short-term benefits, and take the risk of overdose into account.

The safest first step may be to prescribe a selective serotonin reuptake inhibitor. However, the potential for benefit is limited, with anger being the symptom that is most likely helped, at least for a short duration. Another option may be a second-generation antipsychotic agent if cognitive or anger symptoms are primary, but adverse effects of these medications may be substantial, particularly over the long term. Mood stabilizers have some evidence supporting their use as a class for many symptoms; however, evidence to support individual medications comes from only 1 or 2 studies each. Also, the risk of overdose, a primary concern in patients with borderline personality disorder, may be greater with mood stabilizers than with selective serotonin reuptake inhibitors.

Partial responses to medications are common, frequently leading to polypharmacy and treatment for longer than may be necessary. ${ }^{42,50}$ Trying 1 medication at a time is suggested, even in the context of partial response, because there is no evidence to support polypharmacy. Frequent reevaluation of the benefits of any medication is suggested, and prescribers should stop medica- tions when possible. Regardless of the medication used, patients should be fully informed of the limited potential for benefit and the importance of psychotherapy. Overall, pharmacotherapy may be helpful primarily in reducing symptoms sufficiently for patients to stay in psychotherapy. Box 2 provides an example of how this approach may work in practice.

\section{Conclusion}

The treatment of borderline personality disorder is challenging. The past 2 decades have seen the development of specialized psychotherapy techniques designed for this population. These treatments offer effective options that can lead to reductions in self-harm, suicidality and hospital admissions and to overall improvements in symptoms associated with the disorder. Availability of these therapies offers options other than hospital admission. The role of pharmacotherapy remains limited to symptom reduction, although some medications show promise that would require further research to corrob orate. In general, patients with borderline personality disorder now have far more treatment options than they did 2 decades ago; however, the availability of specialized psychotherapy techniques remains limited.

\section{Box 2: Applying the results of this review in clinical practice} (fictional case)

A 23-year-old female university student was brought to the emergency department following a suicide attempt by overdose, having taken medications she had left over from numerous past prescriptions. The suicide attempt was preceded by an argument with a friend she had been chatting with online. The patient had been seen in the emergency department several times over the past 5 years with multiple symptoms, including episodes of depressed mood for several days to weeks following breakups, impulsive suicide attempts, cutting behaviours and transient paranoid symptoms. Over the same time, she had been followed by several clinicians, including community psychotherapists and psychiatrists from different hospitals. She would frequently stop attending psychotherapy or follow-up care, stating that the medications only helped briefly and that she had difficulty connecting with her therapist.

After being assessed in the emergency department, the patient was referred to a 6-month specialized program for the treatment of personality disorders. The program included weekly individual and group sessions that focused on learning to identify her emotional state and those of others, to tolerate unpleasant emotions and to develop techniques to manage interpersonal conflicts. Practical help encouraged the patient to "get a life" by returning to school, build new friendships and develop new pastimes. During her time in the program, most of her medications were stopped, but she remained on a selective serotonin reuptake inhibitor at the end of treatment to "take the edge off." At follow-up 5 years later, she reported having the same job and working full time. She had 2 intimate relationships that did not work out and was now living alone. She said that she still felt sad and lonely at times, with occasional impulsive behaviours, but maintained contact with several friends from school and work. She was not cutting herself, and her suicidal ideation was much less frequent. She was no longer taking medication and was not currently in psychotherapy. 


\section{References}

1. Paris J. Estimating the prevalence of personality disorders in the community. J Personal Disord 2010;24:405-11.

2. Widiger TA, Weissman MM. Epidemiology of borderline personality disorder. Hosp Community Psychiatry 1991;42:1015-21.

3. Zimmerman M, Rothschild L, Chelminski I. The prevalence of DSM-IV personality disorders in psychiatric outpatients. Am J Psychiatry 2005;162:1911-8.

4. Gross R, Olfson M, Gameroff M, et al. Borderline personality disorder in primary care. Arch Intern Med 2002;162:53-60.

5. Sansone RA, Sansone LA, Wiederman MW. Borderline personality disorder and health care utlization in a primary care setting. South Med J 1996;89:1162-5.

6. Hueston WJ, Mainous AG III, Schilling R. Patients with personality disorders: functional status, health care utilization, and satisfaction with care. J Fam Pract 1996;42:54-60.

7. Paris J. Personality disorders over time: precursors, course and outcome. J Pers Disord 2003;17:479-88.

8. Zanarini MC, Frankenburg FR, Reich DB, et al. Time to attainment of recovery from borderline personality disorder and stability of recovery: a 10-year prospective follow-up study. Am J Psychiatry 2010;167:663-7.

9. Paris J, Zweig-Frank H. A 27-year follow-up of patients with borderline personality disorder. Compr Psychiatry 2001;42:482-7.

10. Linehan M. Cognitive-behavioral treatment of borderline personality disorder. New York (NY): Guilford Press; 1993. p. 558.

11. Livesley WJ. Practical management of personality disorder. New York (NY): Guilford Press; 2003. p. 420.

12. Paris J. Treatment of borderline personality disorder: a guide to evidence-based practice. New York (NY): Guilford Press; 2008. p. 260.

13. Dawson DFL, MacMillan HL. Relationship management of the borderline patient: from understanding to treatment. New York (NY): Brunner/Mazel; 1993. p. 212.

14. Boggild AK, Heisel MJ, Links PS. Social, demographic, and clinical factors related to disruptive behaviour in hospital. Can J Psychiatry 2004;49:114-8.

15. Soliman AE, Reza H. Risk factors and correlates of violence among acutely ill adult psychiatric inpatients. Psychiatr Serv 2001;52:75-80

16. Bohus M, Haaf B, Stiglmayr C, et al. Evaluation of inpatient dialectical-behavioral therapy for borderline personality disorder - a prospective study. Behav Res Ther 2000;38:875-87.

17. Bohus M, Haaf B, Simms T, et al. Effectiveness of inpatient dialectical behavioral therapy for borderline personality disorder: a controlled trial. Behav Res Ther 2004;42:487-99.

18. Bateman A, Fonagy P. Mentalization-based treatment for borderline personality disorder: a practical guide. Oxford (UK): Oxford University Press; 2006. p. 188.

19. Clarkin JF, Levy KN, Lenzenweger MF, et al. Evaluating three treatments for borderline personality disorder: a multiwave study. Am J Psychiatry 2077;164:922-8.

20. Giesen-Bloo J, van Dyck R, Spinhoven P, et al. Outpatient psychotherapy for borderline personality disorder: randomized trial of schema-focused therapy vs transference-focused psychotherapy. Arch Gen Psychiatry 2006;63:649-58 (erratum in 2006;63:1008).

21. Weinberg I, Ronningstam E, Goldblatt MJ, et al. Common factors in empirically supported treatments of borderline personality disorder. Curr Psychiatry Rep 2011;13:60-8.

22. Binks CA, Fenton M, McCarthy L, et al. Psychological therapies for people with borderline personality disorder. Cochrane Database Syst Rev 2006; (1):CD005652.

23. Linehan MM, Armstrong HE, Suarez A, et al. Cognitivebehavioral treatment of chronically parasuicidal borderline patients. Arch Gen Psychiatry 1991;48:1060-4.

24. Linehan MM, Tutek DA, Heard HL, et al. Interpersonal outcome of cognitive behavioral treatment for chronically suicidal borderline patients. Am J Psychiatry 1994;151:1771-6.

25. Linehan MM, Heard HL, Armstrong HE. Naturalistic follow-up of a behavioral treatment for chronically parasuicidal borderline patients. Arch Gen Psychiatry 1993;50:971-4 [erratum 1994;51: 422].

26. Verheul R, Van Den Bosch LMC, Koeter MWJ, et al. Dialectical behaviour therapy for women with borderline personality disorder: 12-month, randomised clinical trial in The Netherlands. $B$ J Psychiatry 2003;182:135-40.

27. Carter GL, Willcox CH, Lewin TJ, et al. Hunter DBT project: randomized controlled trial of dialectical behaviour therapy in women with borderline personality disorder. Aust N Z J Psychiatry 2010;44:162-73.

28. Linehan MM, Comtois KA, Murray AM, et al. Two-year randomized controlled trial and follow-up of dialectical behavior therapy vs. therapy by experts for suicidal behaviors and borderline personality disorder. Arch Gen Psychiatry 2006;63:757-66.
29. McMain SF, Links PS, Gnam WH, et al. A randomized trial of dialectical behavior therapy versus general psychiatric management for borderline personality disorder. Am J Psychiatry 2009; 166:1365-74.

30. Soler J, Pascual JC, Tiana T, et al. Dialectical behaviour therapy skills training compared to standard group therapy in borderline personality disorder: a 3-month randomised controlled clinical trial. Behav Res Ther 2009;47:353-8.

31. Stanley B, Brodsky B, Nelson JD, et al. Brief dialectical behavior therapy (DBT-B) for suicidal behavior and non-suicidal self injury. Arch Suicide Res 2007;11:337-41.

32. Linehan MM, Schmidt H III, Dimeff LA, et al. Dialectical behavior therapy for patients with borderline personality disorder and drug-dependence. Am J Addict 1999;8:279-92.

33. Linehan MM, Dimeff LA, Reynolds SK, et al. Dialectical behavior therapy versus comprehensive validation therapy plus 12-step for the treatment of opioid dependent women meeting criteria for borderline personality disorder. Drug Alcohol Depend 2002;67:13-26.

34. Kliem S, Kroger C, Kosfelder J. Dialectical behavior therapy for borderline personality disorder: a meta-analysis using mixedeffects modeling. J Consult Clin Psychol 2010;78:936-51.

35. Bateman A, Fonagy P. Psychotherapy for borderline personality disorder: mentalization-based treatment. Oxford (UK): Oxford University Press; 2004. p. 381.

36. Bateman A, Fonagy P. Randomized controlled trial of outpatient mentalization-based treatment versus structured clinical management for borderline personality disorder. Am J Psychiatry 2009; 166:1355-64.

37. Bateman A, Fonagy P. Effectiveness of partial hospitalization in the treatment of borderline personality disorder: a randomized controlled trial. Am J Psychiatry 1999;156:1563-9.

38. Bateman A, Fonagy P. Treatment of borderline personality disorder with psychoanalytically oriented partial hospitalization: an 18-month follow-up. Am J Psychiatry 2001;158:36-42.

39. Bateman A, Fonagy P. 8-year follow-up of patients treated for borderline personality disorder: mentalization-based treatment versus treatment as usual. Am J Psychiatry 2008;165:631-8.

40. Newton-Howes G, Tyrer P, Johnson T. Personality disorder and the outcome of depression: meta-analysis of published studies. Br J Psychiatry 2006;188:13-20.

41. Zanarini MC, Frankenburg FR, Hennen J, et al. Axis I comorbidity in patients with borderline personality disorder: 6-year follow-up and prediction of time to remission. Am J Psychiatry 2004;161:2108-14.

42. Zanarini MC, Frankenburg FR, Khera GS, et al. Treatment histories of borderline inpatients. Compr Psychiatry 2001;42:144-50.

43. Nosé M, Cipriani A, Biancosino B, et al. Efficacy of pharmacotherapy against core traits of borderline personality disorder: meta-analysis of randomized controlled trials. Int Clin Psychopharmacol 2006;21:345-53.

44. Mercer D, Douglass AB, Links PS. Meta-analyses of mood stabilizers, antidepressants and antipsychotics in the treatment of borderline personality disorder: effectiveness for depression and anger symptoms. J Pers Disord 2009;23:156-74.

45. Ingenhoven $\mathrm{T}$, Lafay $\mathrm{P}$, Rinne $\mathrm{T}$, et al. Effectiveness of pharmacotherapy for severe personality disorders: meta-analyses of randomized controlled trials. J Clin Psychiatry 2010;71:14-25.

46. Lieb K, Vollm B, Rucker G, et al. Pharmacotherapy for borderline personality disorder: Cochrane systematic review of randomised trials. Br J Psychiatry 2010;196:4-12.

47. Zanarini MC, Schulz SC, Detke HC, et al. A dose comparison of olanzapine for the treatment of borderline personality disorder: a 12-week randomized, double-blind, placebo-controlled study. J Clin Psychiatry 2011;72:1358-62.

48. Stoffers J, Vollm BA, Rucker G, et al. Pharmacological interventions for borderline personality disorder. Cochrane Database Syst Rev 2010;(6):CD005653.

49. Paris J. Clinical trials of treatment for personality disorders. Psychiatr Clin North Am 2008;31:517-26

50. Zanarini MC, Frankenburg FR, Hennen J, et al. Mental health service utilization by borderline personality disorder patients and Axis II comparison subjects followed prospectively for 6 years. J Clin Psychiatry 2004;65:28-36.

Affiliations: From the Department of Psychiatry (Biskin, Paris), Institute of Community and Family Psychiatry, Sir Mortimer B. Davis Jewish General Hospital, Montréal, Que.

Contributors: Robert Biskin drafted the manuscript and conducted the literature review. Joel Paris supervised the process and rewrote and edited sections of the manuscript. Both authors approved the final version submitted for publication. 